Clemson University

TigerPrints

$11-2016$

\title{
A Real-Time Programmable Pulsatile Flow Pump for In Vitro Cardiovascular Experimentation
}

Rahul Raj Mechoor

Tyler Schmidt

Ethan Kung

Follow this and additional works at: https://tigerprints.clemson.edu/bioengineering_pubs

Part of the Biomedical Engineering and Bioengineering Commons 


\title{
A REAL-TIME PROGRAMMABLE PULSATILE FLOW PUMP FOR IN-VITRO CARDIOVASCULAR EXPERIMENTATION
}

\author{
Rahul Raj Mechoor \\ Clemson University \\ 252 Fluor Daniel EIB \\ Clemson University \\ Clemson, SC, 29631 \\ rmechoo@clemson.edu \\ Tyler Schmidt \\ Clemson University \\ 252 Fluor Daniel EIB \\ Clemson University \\ Clemson, SC, 29631 \\ tmschmi@clemson.edu \\ ASME Student Member \\ Ethan Kung $^{1}$ \\ Clemson University \\ 231 Fluor Daniel EIB \\ Clemson University \\ Clemson, SC 29634-0921 \\ ekung@clemson.edu \\ ASME Member
}

\section{Citation:}

Mechoor R, Schmidt T, Kung E. "A Real-Time Programmable Pulsatile Flow Pump For In-Vitro Cardiovascular Experimentation." Journal of Biomechanical Engineering. 138(11):111002 (2016)

https://doi.org/10.1115/1.4034561

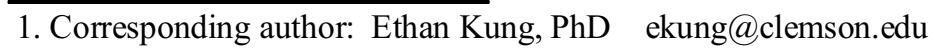


Journal of Biomechanical Engineering

\section{ABSTRACT}

Benchtop In-vitro experiments are valuable tools for investigating the cardiovascular system and testing medical devices. Accurate reproduction of the physiologic flow waveforms at various anatomic locations is an important component of these experimental methods. This study discusses the design, construction, and testing of a low-cost and fully programmable pulsatile flow pump capable of continuously producing unlimited cycles of physiologic waveforms. It consists of a gear pump actuated by an AC servo-motor and a feedback algorithm to achieve highly-accurate reproduction of flow waveforms for flow rates up to $300 \mathrm{ml} / \mathrm{s}$ across a range of loading conditions. The iterative feedback algorithm uses the flow error values in one iteration to modify the motor control waveform for the next iteration to better match the desired flow. Within 4-7 iterations of feedback, the pump replicated desired physiologic flow waveforms to within $2 \%$ normalized RMS error (for flow rates above $20 \mathrm{~mL} / \mathrm{s}$ ) under varying downstream impedances. This pump device is significantly more affordable ( $10 \%$ of the cost) than current commercial options. More importantly, the pump can be controlled via common scientific software and thus easily implemented into large automation frameworks. 
Journal of Biomechanical Engineering

\section{INTRODUCTION}

Cardiovascular diseases are the leading causes of death around the world. According to the American Heart Association, more than 7.6 million cardiovascular procedures were performed in the US with the total treatment spending exceeding $\$ 320$ billion in 2011 [1]. One of the challenges to studying cardiovascular biomechanics is that important quantities such as three dimensional blood flow velocities, as well as stress and strain in blood vessels [2] are difficult to measure clinically. Ethical considerations also present challenges to progress since extensive data collection directly in patients is undesirable. Engineering approaches such as benchtop in-vitro experiments or computational simulations have been used where in-vivo conditions can be recreated for detailed investigation. While computational methods can offer detailed quantifications of hemodynamic parameters, modeling of complex fluid-structure interactions such as device implementation remains challenging. Experimental methods offer the ability to recreate physical interactions that are currently difficult for computational methods to model, and serve as useful platforms for biomechanics investigation and medical device testing.

Benchtop cardiovascular experiments typically utilize medical imaging data and fluid mechanics to recreate physiologically realistic anatomy, pressure, and flow. The flow source, usually a positive displacement pump, creates a pulsatile flow waveform which then feeds into downstream impedances. The downstream impedances typically consist of flow resistors and compliance chambers to mimic vascular impedance, and result in realistic pressure waveforms. One approach to in-vitro experiments is to use a pump to recreate cardiac flow (i.e. the flow through the aortic valve), and a downstream 
Journal of Biomechanical Engineering

vascular simulator, which recreates the impedances along the whole arterial tree, to obtain the desired flow and pressure waveforms at specific anatomic locations. In this approach the flow source produces flow waveforms similar to that in the ascending aorta with varying period lengths. Commercial products, such as the 'Harvard pump' (Harvard Apparatus, Holliston. MA, USA) and the 'SuperPump' (Vivitro Labs, Victoria, BC, Canada), are designed to operate as flow sources in this approach $[3,4]$. These devices offer some level of programmability but the waveforms they can produce are limited to those resembling cardiac flow waveforms with regions of zero flow. This limitation is due to the necessity of these piston pumps to refill the cylinder in each cardiac cycle. The main disadvantage of this approach is the complexity of the required vascular simulator, and that specific localized flow waveforms are difficult to obtain without extensive tuning of the vascular simulator setup. An alternative approach uses a programmable flow source, such as the 'CardioFlow' device (Shelley Medical Imaging Technologies, London, ON, Canada), that can recreate localized flow waveforms for a specific anatomic location[5], thereby eliminating the need for a vascular simulator. However, "CardioFlow" is currently the only available device for this application and it has several drawbacks. First, the output flow waveform may be affected by the varying downstream loading conditions which results in imprecise waveform reproduction. Second, the pump must be controlled by a dedicated computer and stand-alone software, which inhibits direct communication with other devices or programs. Third, with the price of a single unit ranging from $\$ 15,000$ to $\$ 40,000$ (depending on the peak flow rate), cost is a prohibitive factor, especially in experiments that require multiple flow sources. 
Journal of Biomechanical Engineering

Several previous studies have attempted to design a flow pump to reliably reproduce a wide range of localized physiological waveforms with high accuracy while maintaining low cost and complexity. Frayne et al. [6] designed a piston pump capable of flow rates up to $30 \mathrm{~mL} / \mathrm{s}$. Hoskins et al. [7] designed a setup with a gear pump connected to a stepper motor capable of limited flow ranges $(<20 \mathrm{ml} / \mathrm{s})$. A progressive cavity pump was used by Eriksson et al. [8] to produce uninterrupted physiologic waveforms and results were shown for the reproduction of a carotid waveform. Tsai et al. [9] reported results for a carotid waveform from a more complex setup consisting of a gear pump producing constant flow, a piston pump producing the oscillating component of the desired flow, and a back pressure valve to avoid reverse flow into the gear pump. Almost all of these pumps worked under a limited operating range in terms of peak flow, few successfully demonstrated the capability of producing a physiological waveform exhibiting portions of backflow, and most did not have comprehensive characterization data to demonstrate the pump's ability to function in a physiologically realistic experimental setup. Also, there remains the need for a programmable pulsatile flow pump that can be implemented into large automation frameworks. One of the main requirements for such a pump would be the ability to communicate with programs written in common commercial software packages and the ability to change the output flow waveform in real time with minimal human intervention.

The aim of this study is to design, construct and comprehensively test a simple, fully programmable pulsatile flow pump that can consistently and accurately reproduce a wide range of localized physiologic flow waveforms. We performed experiments to characterize pump performance parameters such as peak flow, acceleration and accuracy, 
Journal of Biomechanical Engineering

as well as to demonstrate the pump's capability to recreate flow waveforms in physiologically realistic conditions. The pump is designed to integrate well with commercial software to facilitate automation. The simplicity, affordability, performance, and full-controllability of this design will make it an attractive option for researchers constructing in-vitro cardiovascular experiments.

\section{METHODS}

The flow pump developed in this study uses a gear pump - servo motor combination (Fig. 1). A 40\% glycerin solution is used as the working fluid since it closely approximates the density and viscosity of human blood. The gear pump (Dayton 4KHH8, Grainger Inc.) has a rated maximum continuous output flow rate of $302 \mathrm{ml} / \mathrm{s}$. All materials used in the pump are selected to avoid reactivity with the working fluid. The gear pump is a positive displacement pump which means that the output flow is minimally affected by downstream pressure. Compared to rotodynamic pumps, gear pumps exhibit smaller variations in output flow under varying downstream loading conditions. Gear pumps can also generate negative flows by changing the motor's direction of rotation. Unlike piston pumps, they can be operated continuously and do not need complex tubing connections or valves for switching flow directions. Compared to custom made piston pumps in previous studies, the gear pump's reliability is also superior since it is designed to operate in harsh industrial conditions for long periods of time.

We used a triple pole servo motor (AKM42E-ANCNC-00, Kollmorgen Corporation, Radford, VA, USA) with a rated torque of $3.32 \mathrm{Nm}$ at $4000 \mathrm{rpm}$. The servo controller accurately tracks the motor motion and is advantageous over stepper motors, 
Journal of Biomechanical Engineering

which are unreliable under higher loading conditions due to the possibility of skipped steps. Servo motors also offer smoother motion compared to stepper motors. The chosen motor produces approximately $0.81 \mathrm{HP}$ at $1725 \mathrm{RPM}$. This offers significant headroom over the pump's operating requirement (up to $300 \mathrm{ml} / \mathrm{s}$ flow at physiologic pressures), and enables the pump to handle waveforms with steep acceleration. The motor is controlled using a servo drive (AKD-P00306-NBAN-0000, Kollmorgen Corporation, Radford, VA, USA) that accepts an analog voltage signal as the control signal. The motor and pump shafts are coupled with a servomotor coupling (Servoclass SC 040, Zero-Max, Inc., Plymouth, MN, USA) to allow for small parallel and angular misalignment while ensuring zero backlash. The total cost, including all parts and machining cost is less than \$3000. Following the methods detailed in this study, we estimate that it would take an experienced engineer approximately 7 hours to setup the hardware and software. This estimate includes time for assembly, machining and writing the code for motor control and signal handling.

The pump testing setup (Fig. 1) consists of a reservoir, tubing, a test section where flow measurements are made, and a downstream resistor-capacitor-resistor (RCR) module. The RCR module consists of proximal and distal resistors (pinch valves) with a compliance chamber (air chamber) in the middle. A 3/4 inch inner diameter high pressure PVC tubing with polyester reinforcement is used to connect the reservoir, pump and test section. The low compliance of this tube minimizes flow waveform distortion while the large inner diameter minimizes flow resistance. The test section has Tygon tubing with 3/8 inch inner diameter for flow measurement. The RCR module [10] is used for creating physiologic pressures. 
Journal of Biomechanical Engineering

Control signal generation and data acquisition is carried out using LABVIEW software package (National Instruments, Austin, TX, USA) through separate modules (NI 9205, NI 9263, National Instruments, Austin, TX, USA) on a compact DAQ chassis (NI cDAQ 9174, National Instruments, Austin, TX, USA). An ultrasonic transit time flow meter (TS 410 with ME-9PXL flow sensor, Transonic Systems Inc., Ithaca, NY) with a low pass filter set at $40 \mathrm{~Hz}$ is used to measure instantaneous flow rates. A pressure transducer (Argon DTX Plus, Argon Medical Devices Inc., Plano, TX, USA) with a pressure control unit (Millar PCU-2000, Millar, Inc., Houston, TX, USA) is used to measure pressure at the pump outlet. Measurements are typically averaged over 5 periods to reduce the effect of sensor noise.

In addition to signal handling, the custom made LABVIEW program incorporates a feedback algorithm that uses a semi-real time iterative process to reduce the error in the output flow. When a desired flow waveform is prescribed, it is processed in a feedback loop until the error in the output waveform is within a user specified limit. This is achieved via a proportional error control that uses the measured error in each iteration to modify the corresponding motor control signal for the next iteration. During each iteration, the error in flow at each individual data point is added to the corresponding data point in the motor speed control waveform for the next iteration to better match the desired flow (equation 1). The error in flow is multiplied by a constant to convert it to motor RPM. This constant is specific to the gear pump used and is found via pump calibration (equation 2).

New motor speed $(R P M)=$ Current motor speed $(R P M)+($ Current error $(\mathrm{ml} / \mathrm{s}) *$ 
Journal of Biomechanical Engineering

A series of tests were conducted to benchmark the pump. An initial flow calibration was performed where the pump was operated at constant speeds ranging from -1400 to 1400 RPM with and without a downstream resistance to check the linearity of the pump output flow with respect to motor RPM and its dependence on downstream conditions. An acceleration test was conducted to ascertain the maximum possible flow acceleration. Then, a feedback test was conducted to evaluate the feedback loop by analyzing the change in output flow waveform after each feedback iteration. Next, we demonstrated the pump's performance in an experiment featuring physiologic flow and pressure waveforms in two different scenarios. The abdominal aortic flow waveform under exercise condition was used to represent a simple, higher flow rate physiologic waveform and the abdominal aortic waveform under resting condition was used to test the capability of the pump to produce complex physiologic waveforms containing backflow. 5 cycles of flow data was averaged to obtain each flow waveform for the feedback test and the physiologic flow test. The cycle-to-cycle uniformity of the output flow waveforms were evaluated by superposing multiple waveforms without averaging and finding the maximum deviation between the cycles.

The reference physiological waveforms used are abdominal aortic flow and pressure waveforms under exercise (Fig. 2.a) and resting conditions (Fig. 2.b) with mean flow rates of $65.4 \mathrm{ml} / \mathrm{s}(3.9 \mathrm{l} / \mathrm{min})$ and $22.8 \mathrm{ml} / \mathrm{s}(1.4 \mathrm{l} / \mathrm{min})$ respectively, as reported previously [11]. The abdominal aortic flow waveform under exercise condition was representative of a forward-only waveform, whereas the resting waveform was 
Journal of Biomechanical Engineering

representative of a waveform with back flow and high frequency, low amplitude oscillations.

\section{RESULTS AND DISCUSSION}

Pump calibration was performed by running the pump at a series of constant speeds from -1400 to $+1400 \mathrm{rpm}$ at intervals of $200 \mathrm{rpm}$ for a period of $5 \mathrm{~s}$ each. The same procedure was repeated with the downstream valve partly closed to induce back pressure up to $165 \mathrm{mmHg}$ (at $170 \mathrm{ml} / \mathrm{s}$ flow rate). Figure 3 shows that the relationship between motor RPM and flow rate is linear within the operating range, and that back pressure has a minimal effect on flow output. The relationship between motor speed and pump output flow rate, shown in equation 2 , was derived from this result and is used to predict the initial control waveform for all desired flow waveforms.

$$
\text { Motor speed }(\text { RPM })=\text { Flow Rate }(\mathrm{ml} / \mathrm{s}) * 7.252
$$

The impulse response of the pump was analyzed to determine the maximum achievable flow acceleration. This value can be used to determine whether the pump can recreate a specific waveform by examining the maximum acceleration present in the waveform. A step waveform from 0 to $190 \mathrm{ml} / \mathrm{s}$ was used for this test. The pump required $0.135 \mathrm{~s}$ to achieve this change in flow rate, implying a maximum acceleration of $1413 \mathrm{ml} / \mathrm{s}^{2}$

The semi-real time feedback algorithm was analyzed by tracking changes in the output abdominal aortic flow waveform under exercise condition in each iteration. Figure 4a shows the desired flow waveform compared with output flow waveform without any feedback and that after 6 iterations of the feedback, with the latter being nearly identical to the desired flow waveform. Figure $4 \mathrm{~b}$ shows that the normalized RMS error generally 
Journal of Biomechanical Engineering

decreases with iterations and converges by around 4-7 iterations. The feedback loop increases the accuracy of the output flow waveform and reduces the error from $6.2 \%$ with no feedback to $0.71 \%$ after 6 feedback iterations. This feedback capability will be especially useful in cases where the waveform may be distorted by a longer or more compliant tubing connecting the pump and test section.

Next, we demonstrate physiologic scenarios featuring realistic abdominal aortic flow and pressure waveforms. An RCR module was used to provide physiologically realistic downstream impedance. The compliance chamber was tuned according to our previous study [11]. The resistances were manually tuned to obtain downstream pressure waveforms similar in shape to those in figure 2 (note that the exact matching of downstream pressure is not the focus of this study). Under realistic downstream pressures, for the exercise waveform the pump accurately reproduced the flow waveform with a normalized RMS error of $1.08 \%$ and an $\mathrm{R}^{2}$ value of 0.9995 (fig. 5a). The abdominal aortic waveform under resting condition was used to assess the ability of the pump to replicate complex waveforms with negative flow regions. The result (fig. 5b) shows that the pump successfully recreated the flow with a normalized RMS error of $2.72 \%$ over the cycle and an $\mathrm{R}^{2}$ value of 0.9997 . This result also demonstrates the pump's capability to produce small amplitude oscillations that could be present in human aortic waveforms.

The cycle-to-cycle consistency of the output flow waveforms is shown in figure 6 by superposing 20 individual waveforms from a series of consecutive waveforms. The deviation in flow rate at each time point was calculated by using the maximum and minimum values of flow across the 20 cycles. The results show a normalized RMS 
Journal of Biomechanical Engineering

deviation of $3.4 \%$ and an $\mathrm{R}^{2}$ value of 0.9895 which implies a high uniformity across waveforms produced in each cycle.

Summarizing these results, Table 1 shows the RMS error, normalized RMS error and the $\mathrm{R}^{2}$ values for all the relevant tests conducted. The quantitative results from these tests show that our pump design performs better than or as good as those in previous studies in all aspects. The peak flow rate of $300 \mathrm{ml} / \mathrm{s}$ is on par with the best $[8$, 9]. The physiologically realistic flow accuracy at $\pm 1 \%$ to $\pm 2 \%$ is better than those in previous studies ( $\pm 1 \%$ to $\pm 15 \%)$ and most commercial systems ( $\pm 2 \%$ to $\pm 4 \%$ ) [3-9]. This pump also has several nontechnical advantages over those in previous studies. It takes less than two minutes from when the desired waveform is specified for the pump to produce an accurate replica. This is significantly shorter time compared to previous studies utilizing feedback. After the initial setup, no additional human intervention is necessary, and the pump can be completely controlled by computational programs. This ability for automation is a useful feature for experimental setups where large numbers of flow waveforms are to be tested in an automated framework. An example of such an experiment is the investigation of flows through the same anatomical location under various levels of exercise conditions and arterial stiffening which affects flow pulse amplitude. Such a study would require testing of a large matrix of different flow conditions, which would benefit significantly from an automation capability. This pump is also considerably more affordable than commercial pumps.

\section{$\underline{\text { Limitations }}$}

Even though the feedback algorithm increases output accuracy, it occasionally causes a distortion of the control waveform due to an accumulation of sensor noise. This 
Journal of Biomechanical Engineering

is solvable by further investigation and modification of the feedback algorithm, as well as averaging of flow measurements across a larger number of cycles. The current pump is optimized for peak flow rates between $50-300 \mathrm{ml} / \mathrm{s}(3-18 \mathrm{l} / \mathrm{min})$. Flow waveforms below $50 \mathrm{ml} / \mathrm{s}$ or above $300 \mathrm{ml} / \mathrm{s}$ should be reproduced by using smaller or larger gear pumps respectively. The current design can be modified to service other ranges of flow rates by switching only the gear pump at a fraction (15\%) of the cost of the whole setup. Even though the pump is theoretically capable of producing a peak flow rate of $300 \mathrm{ml} / \mathrm{s}$, we were only able to confirm the pump performance up to $170 \mathrm{ml} / \mathrm{s}$ due to the limitation of the flow sensor used.

\section{CONCLUSION}

In this study, we successfully designed, built, and comprehensively tested a realtime, fully programmable, pulsatile flow pump that can produce realistic physiologic flow waveforms under physiologic downstream impedances. While being more affordable than current commercial pumps, our design is capable of reproducing flow waveforms containing backflow as well as high frequency oscillations with less than $2 \%$ normalized RMS error (for flow rates above $20 \mathrm{~mL} / \mathrm{s}$ ). The pump can quickly create accurate reproductions of the desired waveform with minimal human interaction. The pump is controlled using commercial software which can be integrated with other computational programs, making it suitable for use in a fully automated framework. Future work will consist of testing the design under wider flow ranges implementing different gear pumps and flow sensors, and improving the feedback algorithm to prevent control waveform divergence due to sensor noise. 
Journal of Biomechanical Engineering

\section{ACKNOWLEDGMENTS}

This study is supported by funding from the Mechanical Engineering department of Clemson University. We also acknowledge Michael Justice, Jamie Cole and Stephen Bass for their assistance in the pump construction.

\section{REFERENCES}

[1] Mozaffarian, D., Benjamin, E. J., Go, A. S., Arnett, D. K., Blaha, M. J., Cushman, M., de Ferranti, S., Despres, J.-P., Fullerton, H. J., Howard, V. J., Huffman, M. D., Judd, S. E., Kissela, B. M., Lackland, D. T., Lichtman, J. H., Lisabeth, L. D., Liu, S., Mackey, R. H., Matchar, D. B., McGuire, D. K., Mohler, E. R., Moy, C. S., Muntner, P., Mussolino, M. E., Nasir, K., Neumar, R. W., Nichol, G., Palaniappan, L., Pandey, D. K., Reeves, M. J., Rodriguez, C. J., Sorlie, P. D., Stein, J., Towfighi, A., Turan, T. N., Virani, S. S., Willey, J. Z., Woo, D., Yeh, R. W., and Turner, M. B., 2014, "Heart Disease and Stroke Statistics--2015 Update: A Report From the American Heart Association", Circulation, 131(4), pp. e29-e322.

[2] Glagov, S., Zarins, C., Giddens, D., and Ku, D., 1988, "Hemodynamics and atherosclerosis. Insights and perspectives gained from studies of human arteries..pdf," Arch. Pathol. Lab. Med., 112(10), pp. 1018-1031.

[3] Groves, E. M., Falahatpisheh, A., Su, J. L., and Kheradvar, A., 2014, "The Effects of Positioning of Transcatheter Aortic Valves on Fluid Dynamics of the Aortic Root," Asaio J, 60(5), pp. 545-552

[4] Pahlevan, N. M., and Gharib, M., 2013, "In-vitro investigation of a potential wave pumping effect in human aorta," J. Biomech., 46(13), pp. 2122-2129.

[5] Kefayati, S., and Poepping, T. L., 2010, "3-D flow characterization and shear stress in a stenosed carotid artery bifurcation model using stereoscopic PIV technique," 2010 Annu. Int. Conf. IEEE Eng. Med. Biol. Soc. EMBC'10, pp. 3386-3389.

[6] Frayne, R., Holdsworth, D. W., Gowman, L. M., Rickey, D. W., Drangova, M., Fenster, A., and Rutt, B. K., 1992, "Computer-controlled flow simulator for MR flow studies.," J. Magn. Reson. Imaging, 2(5), pp. 605-612.

[7] Hoskins, P. R., Anderson, T., and McDicken, W. N., 1989, "A computer controlled flow phantom for generation of physiological Doppler waveforms.," Phys. Med. Biol., 34(11), pp. 1709-1717.

[8] Eriksson, A., Persson, H. W., and Lindstrom, K., 2000, “A computer-controlled arbitrary flow wave form generator for physiological studies," Rev. Sci. Instrum., 71(1), pp. 235-242.

[9] Tsai, W., and Savaş, O., 2010, "Flow pumping system for physiological waveforms.," Med. Biol. Eng. Comput., 48(2), pp. 197-201. 
Journal of Biomechanical Engineering

[10] Kung, E. O., and Taylor, C. A., 2011, “Development of a Physical Windkessel Module to Re-Create In Vivo Vascular Flow Impedance for In Vitro Experiments," Cardiovasc. Eng. Technol., 2(1), pp. 2-14.

[11] Kung, E. O., Les, A. S., Medina, F., Wicker, R. B., McConnell, M. V, and Taylor, C. a, 2011, "In vitro validation of finite-element model of AAA hemodynamics incorporating realistic outlet boundary conditions.," J. Biomech. Eng., 133(4), p. 041003. 
Journal of Biomechanical Engineering

\section{Table Captions List}

Table $1 \quad$ Summary of testing results 
Tables

\begin{tabular}{|c|c|c|c|}
\hline Test parameters & $\begin{array}{c}\text { RMS error in flow } \\
(\mathrm{ml} / \mathrm{s})\end{array}$ & $\begin{array}{c}\text { Normalized RMS } \\
\text { error in flow } \\
(\%)\end{array}$ & $\mathbf{R}^{2}$ Value \\
\hline $\begin{array}{c}\text { Exercise abdominal aortic flow } \\
\text { (Fig 4a) }\end{array}$ & 0.47 & 0.71 & 0.9998 \\
\hline $\begin{array}{c}\text { Exercise abdominal aortic flow without } \\
\text { feedback } \\
\text { (Fig 4a) }\end{array}$ & 4.09 & 6.20 & 0.9848 \\
\hline $\begin{array}{c}\text { Exercise abdominal aortic flow under } \\
\text { physiologic pressure } \\
\text { (Fig 5a) }\end{array}$ & 0.72 & 1.08 & 0.9995 \\
\hline $\begin{array}{c}\text { Resting abdominal aortic flow under } \\
\text { physiologic pressure } \\
\text { (Fig 5b) }\end{array}$ & 0.62 & 2.72 & 0.9997 \\
\hline \begin{tabular}{c} 
(Fig) \\
\hline
\end{tabular}
\end{tabular}

Table 1 Summary of testing results 


\section{Figure Captions List}

Fig. 1 System schematic. (1) 3/4 inch ID reinforced high pressure PVC tube. (2) Pressure sensor. (3) Flow sensor. (4) Pinch valves. (5) Compliance chamber. (6) $3 / 8$ inch ID Tygon tubing

Fig. 2 Physiologic abdominal aortic flow (solid black line) and pressure (dotted blue line) under light exercise (a) and resting (b) conditions [11]

Fig. 3 Calibration curve: Motor Speed vs Flow Rate with downstream valve fully open (blue square) and partially closed (red star)

Fig. 4 Comparison of desired flow (solid black line) with output flow without feedback (dotted blue line) and output flow after 6 iterations of feedback (dotted red line) (a). Convergence of the output flow waveform (b)

Fig. 5 Results from physiological testing. Recreation of abdominal aortic waveform under exercise (a) and resting (b) conditions. Desired flow (solid black line) compared to output flow (dotted red line) and pressure (dotted blue line)

Fig. 6 Twenty individual waveforms from a series of continuous waveforms superposed over each other to show the cycle-to-cycle consistency in output flow 


\section{FIGURES}

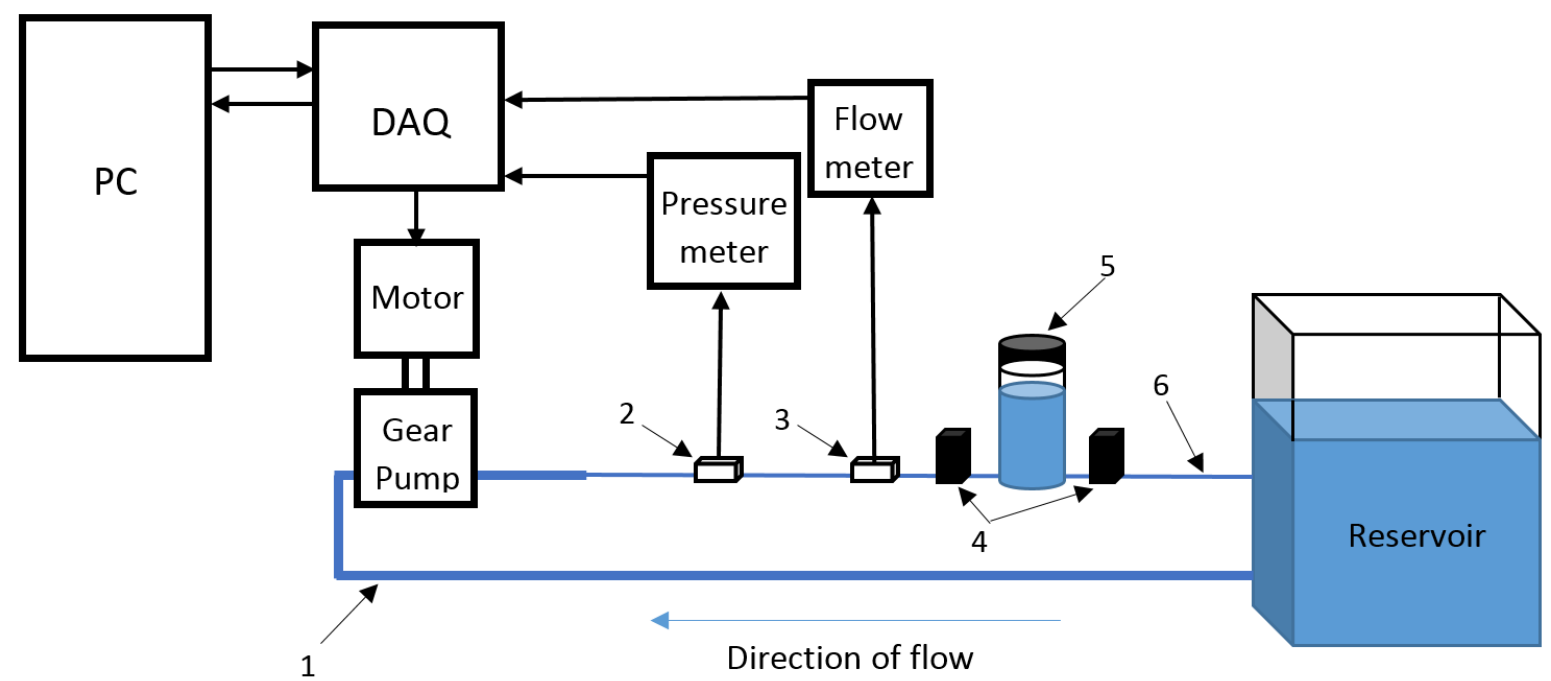

Fig. 1 System Schematic. (1) 3/4 inch ID reinforced high pressure PVC tube. (2) Pressure Sensor. (3) Flow Sensor. (4) Pinch valves. (5) Compliance Chamber. (6) 3/8 inch ID Tygon tubing 
(a)

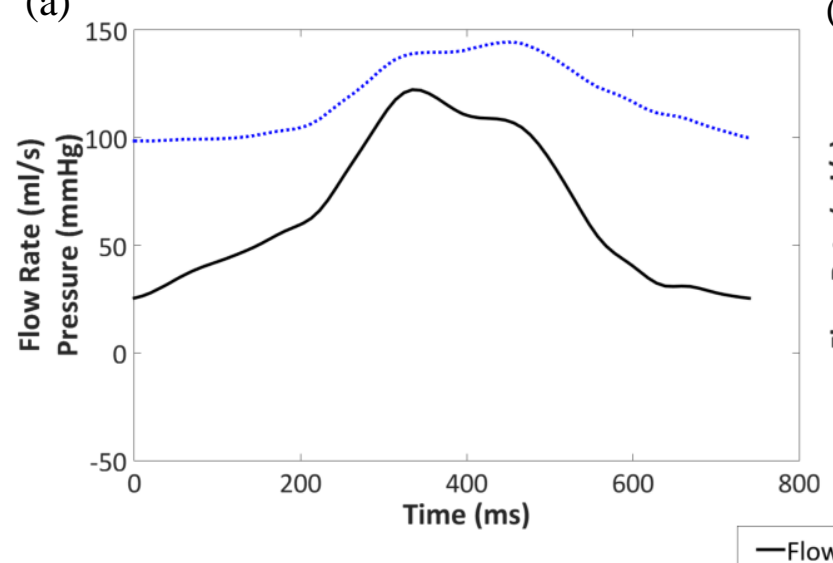

(b)

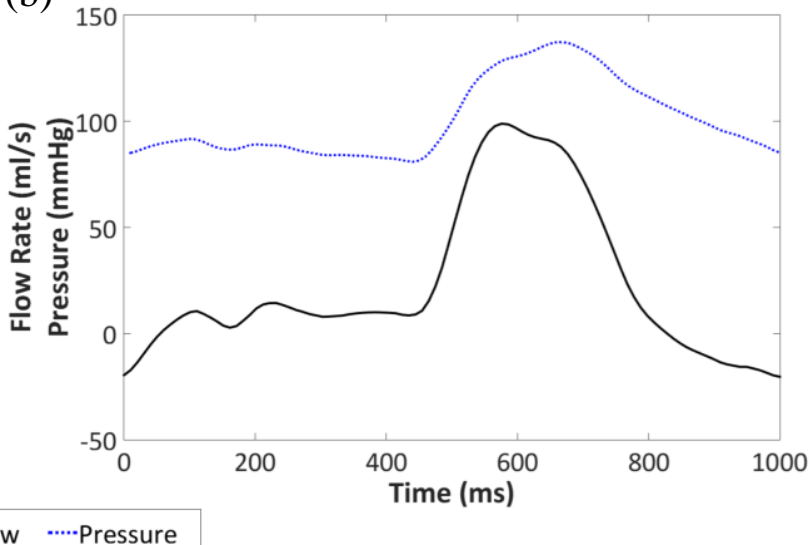

Fig. 2 Physiologic abdominal aortic flow (solid black line) and pressure (dotted blue line) under light exercise (a) and resting (b) conditions [11] 


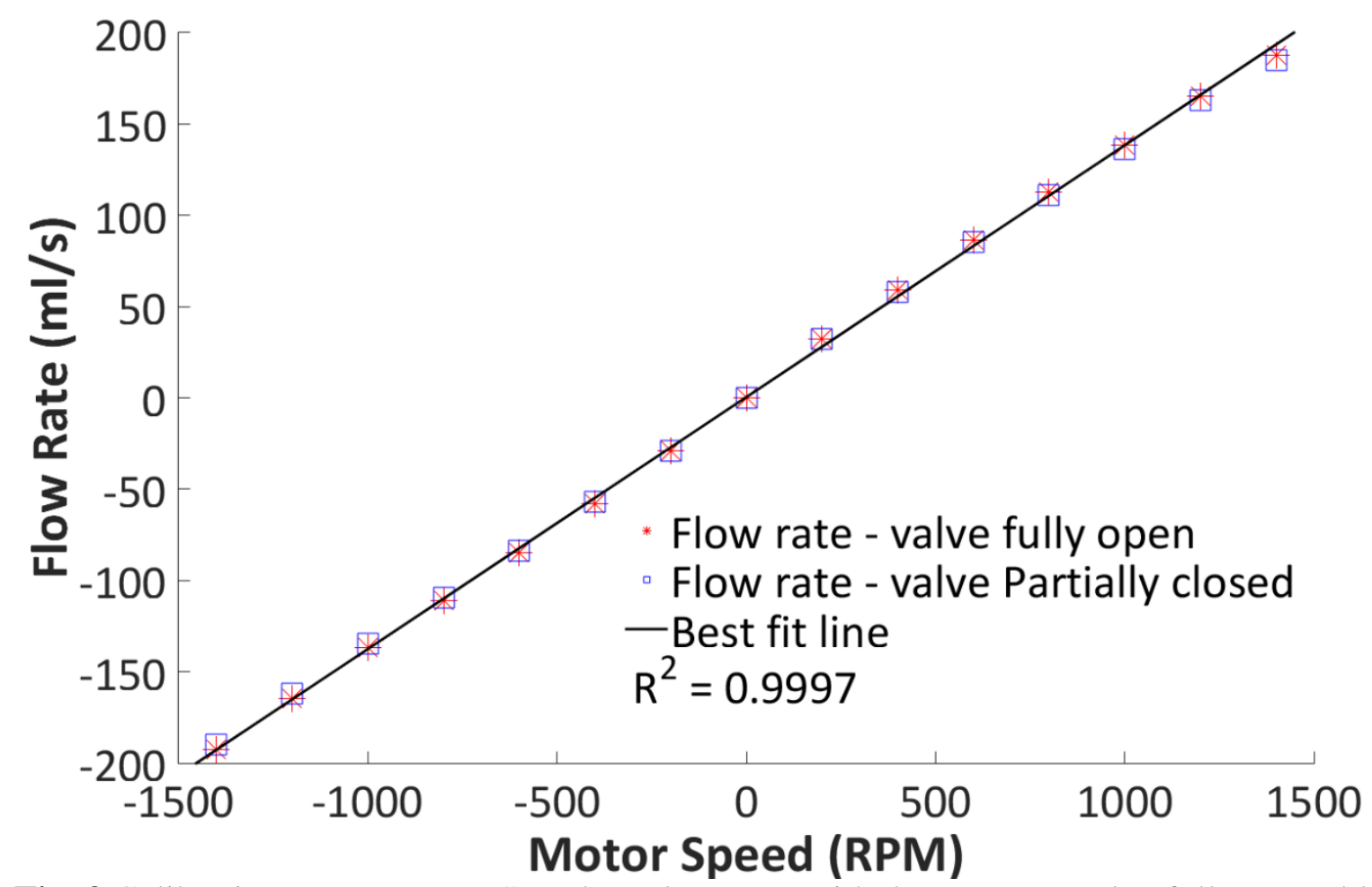

Fig. 3 Calibration curve: Motor Speed vs Flow Rate with downstream valve fully open (blue square) and partially closed (red star) 
(a)

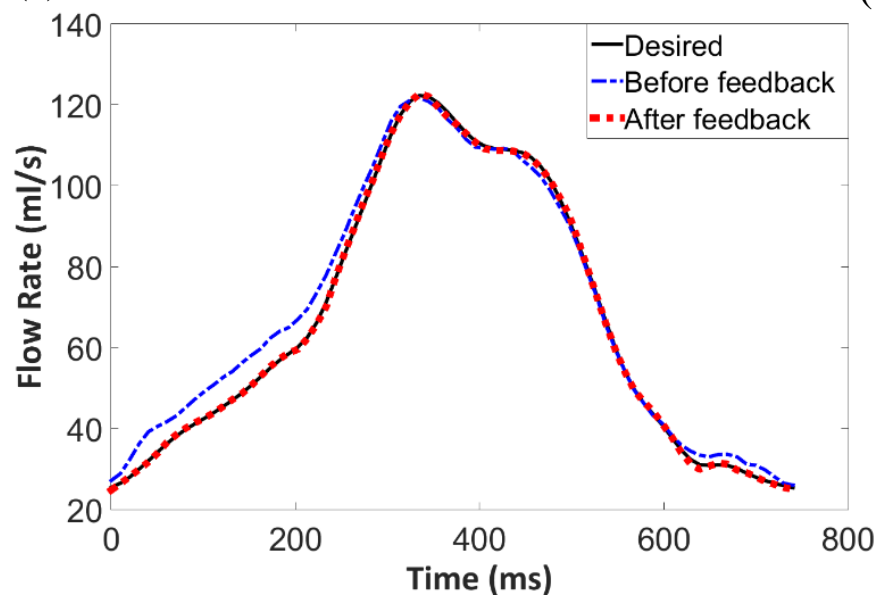

(b)

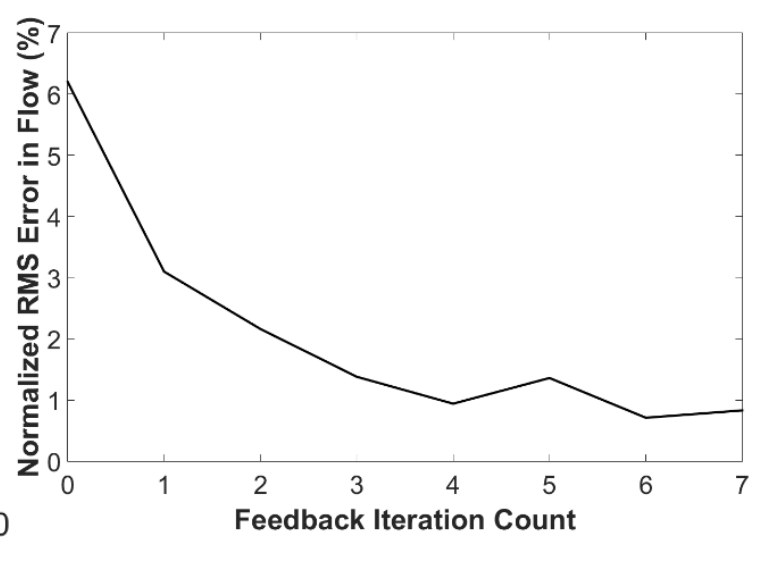

Fig. 4 Comparison of desired flow (solid black line) with output flow without feedback (dotted blue line) and output flow after 6 iterations of feedback (dotted red line) (a). Convergence of the output flow waveform (b) 


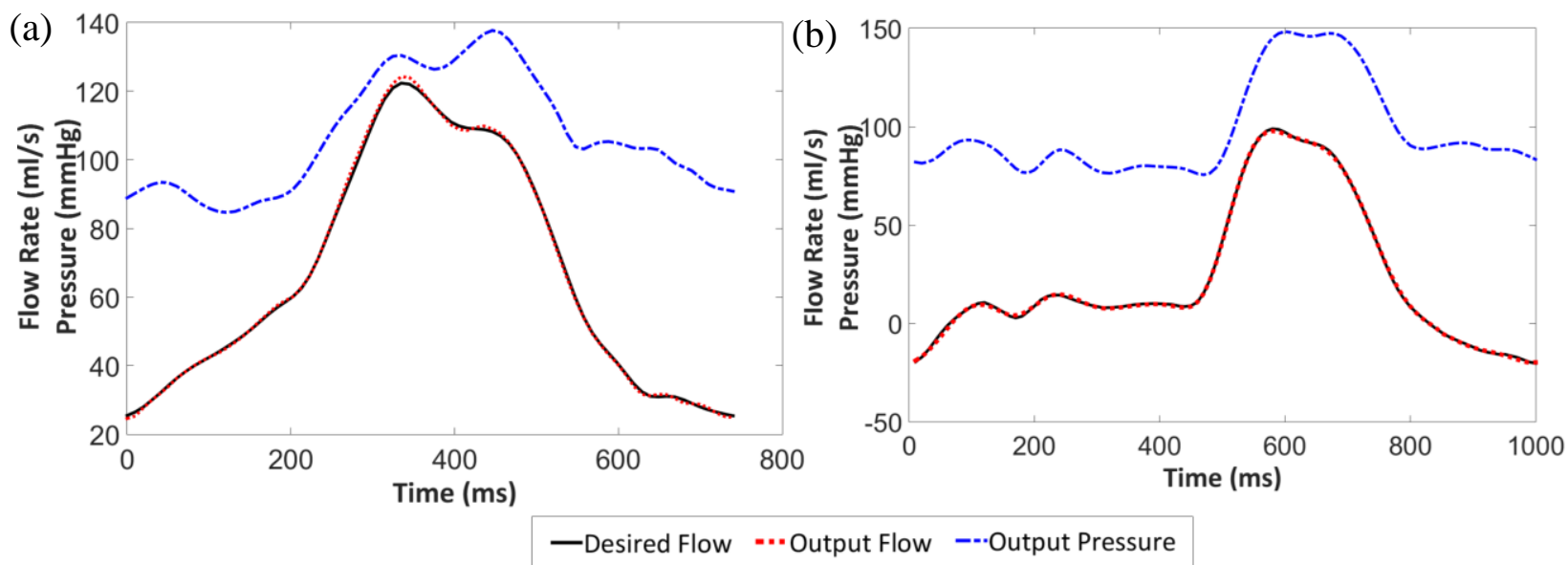

Fig. 5 Results from physiological testing. Recreation of abdominal aortic waveform under exercise (a) and resting (b) conditions. Desired flow (solid black line) compared to output flow (dotted red line) and pressure (dotted blue line) 


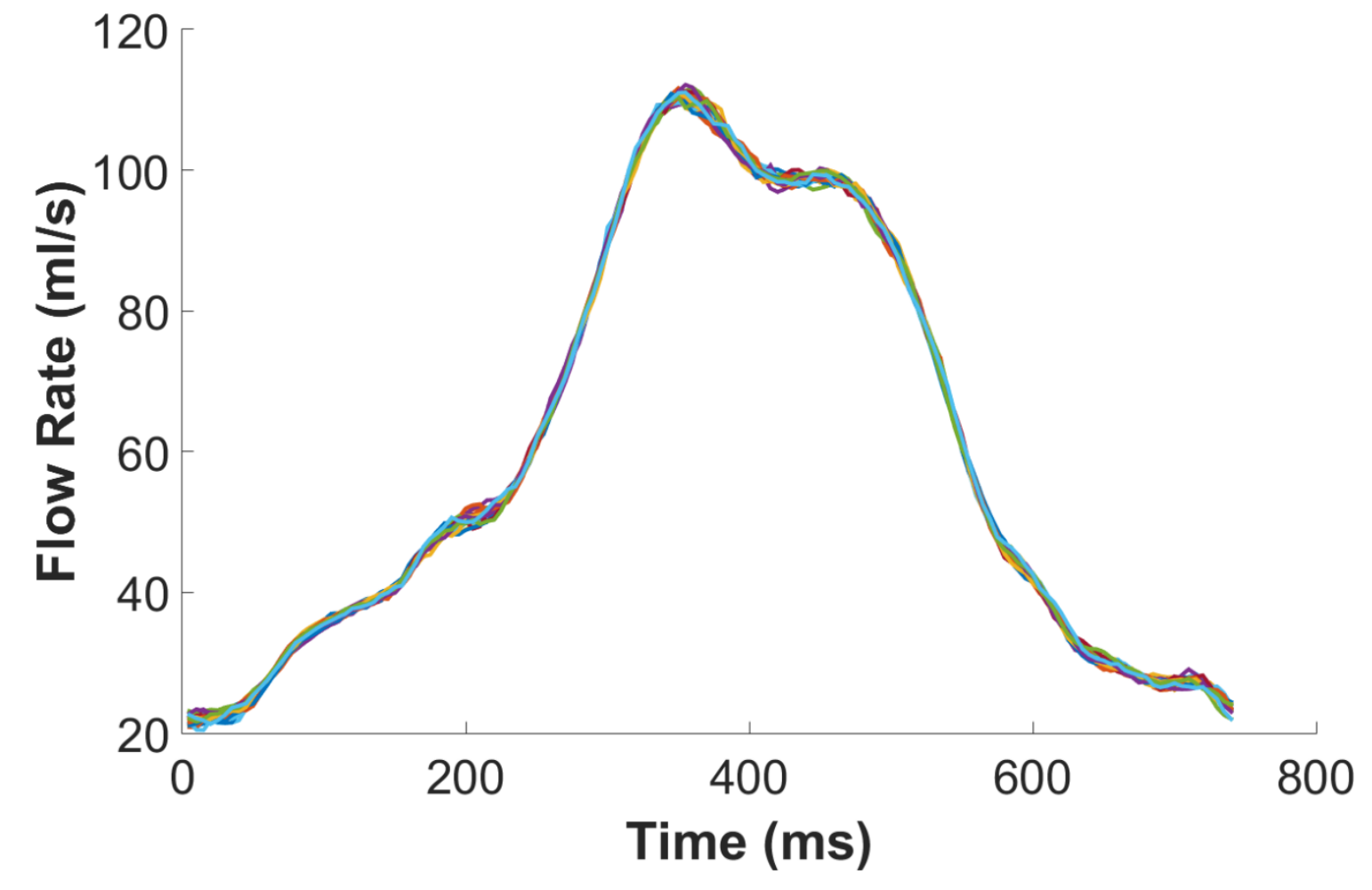

Fig. 6 Twenty individual waveforms from a series of continuous waveforms superposed over each other to show the cycle-to-cycle consistency in output flow 